\title{
The Right Measure and its Consequences
}

It is not just since digitalisation and mobility transformation swept over us that large parts of our economy have been facing change. Successful businesses adapt to changing circumstances - this principle is as old as trade itself. Suppliers have been especially successful at this in recent decades. We can thank supplier companies in particular for the fact that we can today enjoy complex, high-tech products that even in their tiniest components - just think about seals or connectors - are the fruit of years of research. It is no coincidence that suppliers, for example in the German automotive industry, generate $70 \%$ of added value, or $50 \%$ of added value in the global rail vehicle industry. The significance of these hidden champions for our labor market is well known.

I recently listened to a presentation on the future of lightweight design. The speaker called for a new unit of measure to be established for lightweight design: functional weight. Or more scientifically: mass per unit function. This makes sense, as so far we have usually rated lightweight design solutions according to the weight saved in relation to a reference component - for example through the use of lighter materials. And yet how do you assess a structure made from conventional materials but which combines the function of a number of components or systems in a single unit?

If the answer is functional weight, a second question arises: Who has the competence for lightweight design spanning a number of different systems? Who is able to merge individual systems for different functions into a single entity? In the first place, the OEM. Although suppliers produce outstanding individual components, they lack an understanding of the system as a whole, since they are not sufficiently involved in the development of a car or rail vehicle. Does this mean a shift in lightweight design competence in our economy characterised as it is by diversified small and medium enterprises back to the OEMs?

Although it will surely not turn out as bad as that, we should not take the development too lightly. New processes are needed that systematically facilitate weight-optimised products - namely in inter-company projects. While protecting the special knowledge of each project partner, such a process must also be sufficiently transparent to ensure that each supplier understands how their development influences the overall project. 